\title{
Hierarchical Quantized Representations for Script Generation
}

\author{
Noah Weber ${ }^{1}$, Leena Shekhar ${ }^{1}$, Niranjan Balasubramanian ${ }^{1}$, Nathanael Chambers ${ }^{2}$ \\ ${ }^{1}$ Stony Brook University, NY, USA \\ \{nwweber, lshekhar, niranjan\}@cs.stonybrook.edu \\ ${ }^{2}$ United States Naval Academy, MD, USA \\ nchamber@usna. edu
}

\begin{abstract}
Scripts define knowledge about how everyday scenarios (such as going to a restaurant) are expected to unfold. One of the challenges to learning scripts is the hierarchical nature of the knowledge. For example, a suspect arrested might plead innocent or guilty, and a very different track of events is then expected to happen. To capture this type of information, we propose an autoencoder model with a latent space defined by a hierarchy of categorical variables. We utilize a recently proposed vector quantization based approach, which allows continuous embeddings to be associated with each latent variable value. This permits the decoder to softly decide what portions of the latent hierarchy to condition on by attending over the value embeddings for a given setting. Our model effectively encodes and generates scripts, outperforming a recent language modeling-based method on several standard tasks, and allowing the autoencoder model to achieve substantially lower perplexity scores compared to the previous language modelingbased method.
\end{abstract}

\section{Introduction}

Scripts were originally proposed by Schank and Abelson (1977) as "structures that describe the appropriate sequence of events in a particular context". These event sequences define expectations for how common scenarios (such as going to a restaurant) should unfold, thus enabling better language understanding. Although scripts represented many other factors (roles, entry conditions, outcomes) recent work in script induction (Rudinger et al., 2015; Pichotta and Mooney, 2016; Peng and Roth, 2016) has focused on language modeling (LM) approaches where the "appropriate sequence of events" is the textual order of events (tuples of event predicates and their arguments). Modeling a distribution of text se-

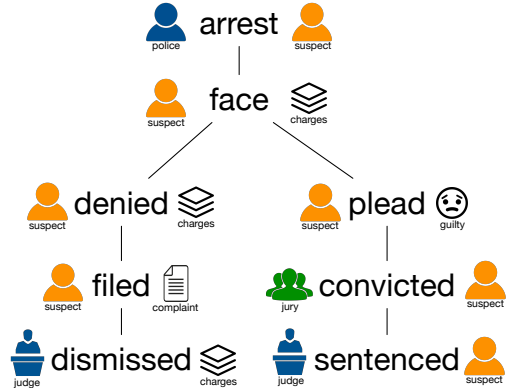

Figure 1: An automatically learned multi-track script. The left track is a dismissed case, and the right is a convicted suspect. Our model generated both tracks through a latent hierarchy.

quences gives the intuitive interpretation of appropriate event sequences being roughly equivalent to probable textual sequences. We continue with an LM approach, but we tackle two very important LM problems that have not yet been addressed with regards to event sequence modeling.

The first problem to address is that language models tend towards local coherency. Count based models are restricted by window size and sparse counts, while neural language models are known to rely on the local context for predictions. Since scripts are meant to describe longer coherent scenarios, this is a major issue. For example, contradictory events like (he denied charges) and (he pleads guilty) are given high probability in a typical language model. Our model instead captures these variations with learned latent variables.

The second problem with recent work is that the hierarchical nature of scripts is not explicitly captured. A high level script (like a suspect getting arrested) can branch off into many possible variations. These variations are called the "tracks" of a script. Figure 1 shows a script with two tracks learned by our model. LM-based approaches often fail to explicitly capture this structure, instead 
throwing it all into one big distribution. This muddies the water for language understanding, making it difficult to tease apart differences like going to a fancy restaurant or a casual restaurant.

To remedy these problems, we propose a model that captures hierarchical structure via global latent variables. The latent variables are categorical (representing the various types of scripts and thier possible tracks and variations) and form a tree (or more generally, a DAG) ${ }^{1}$, thus capturing hierarchical structure with the top (or bottom) levels of the tree representing high (or low) level features of the script. The top might control for large differences like restaurant vs crime, while the bottom selects between fancy and casual dining.

The overall model, which we describe below, takes the form of an autoencoder, with an encoder network inferring values of the latents and a decoder conditioned on the latents generating scripts. We show the usefulness of these latent representations against a prior RNN language model based system (Pichotta and Mooney, 2016) on several tasks. We additionally evaluate the perplexity of the system against the RNN language model, a task that autoencoder models have typically struggled with (Bowman et al., 2016). We find that the latent tree reduces model perplexity by a significant amount, possibly indicating the usefulness of the model in a more general sense.

\section{Background}

\subsection{Variational Autoencoders}

Variational Autoencoders (VAEs, Kingma and Welling (2014)) are generative models which learn latent codes $z$ for the data $x$ by maximizing a lower bound on the data likelihood:

$$
\log (p(x)) \geq \mathrm{E}_{q(z \mid x)}[p(x \mid z)]-K L[q(z \mid x) \| p(z)]
$$

VAEs consist of two components: an encoder which parameterizes the latent posterior $q(z \mid x)$ and a decoder which parameterizes $p(x \mid z)$. The objective function can be made completely differentiable via the reparameterization trick, with the full model resembling an autoencoder and the KL term acting as a regularizer.

While VAEs have been useful in continuous domains, they have been less successful in generating discrete domains whose outputs have lo-

\footnotetext{
${ }^{1}$ In this work we only look at linear chains of categorical variables, which is enough to encode trees (such as the one in Figure 1)
}

cal syntactic regularities. Part of this is due to the "posterior collapse" problem (Bowman et al., 2016); when VAEs are equipped with powerful autoregressive decoders, they tend to ignore the latent, collapsing the posterior $q(z \mid x)$ to the (usually zero-mean Gaussian) prior $p(z)$. By doing this, the model takes no penalty from the KL term, but effectively ignores its encoder.

\subsection{Vector Quantized Variational Autoencoders}

Vector Quantized VAEs (VQ-VAEs, van den Oord et al. (2017)) are a recently proposed class of models which both alleviates the posterior collapse problem and allows the model to use a latent space of discrete values. In VQ-VAEs the latent $z$ is represented as a categorical variable that can take on $K$ values. Each of these values $k \in\{1, \ldots, K\}$ has associated with it a vector embedding $e_{k}$. The posterior of VQ-VAEs are discrete, deterministic, and parameterized as follows:

$$
q(z=k \mid x)= \begin{cases}1 & \mathrm{k}=\operatorname{argmin}_{i}\left\|f(x)-e_{i}\right\|_{2} \\ 0 & \text { elsewise }\end{cases}
$$

where $f(x)$ is a function defined by an encoder network. The decoding portion of the network is similar to VAEs, where a decoder parameterizes a distribution $p(x \mid z=k)=g\left(e_{k}\right)$, where $g$ is the decoder network, and $e_{k}$ is the corresponding embedding, which is fed as input to the decoder. This process can be seen as a "quantization" operation mapping the continuous encoder output to the latent embedding it falls closest to, and then feeding this latent embedding (in lieu of the encoder output) to the decoder.

The quantization operator is not differentiable, thus during training, the gradient of the loss with respect to the decoder input is used as an estimation to the gradient of the loss with respect to the encoder output. If one assumes a uniform prior over the latents (we do so here), then the KL term in the VAE objective becomes constant and may be ignored. In practice, multiple latent variables $z$ may by used, each with their own (or shared) embeddings space.

\section{Hierarchical Quantized Autoencoder}

Our goal is to build a model that can generate globally coherent multi-track scripts which allow us to account for the different ways in which a script can unfold. The main idea behind our approach 




Figure 2: Hierarchical Quantized Autoencoder Architecture

is to use a hierarchical latent space which conditions the generation of the scripts. The VQ-VAE models (described earlier) provide a way to model discrete variables in an autoencoding framework while avoiding the posterior collapse problem. We build on this framework to propose a new HierarchicAl Quantized Autoencoder (HAQAE) model.

HAQAEs are autoencoders with $M$ latent variables, $z_{0}, \ldots, z_{M}$. Each latent variable is categorical taking on $K$ different values. Like in VQVAEs, every categorical value $k$ for variable $z$ has an associated embeddings $e_{z k}$. The latent variables are given a tree structure and the full posterior over all $M$ latents $\mathbf{z}$ factorizes as:

$$
q(\mathbf{z} \mid x)=q_{0}\left(z_{0} \mid x\right) \prod_{i=1}^{M-1} q_{i}\left(z_{i} \mid \operatorname{pr}\left(z_{i}\right), x\right)
$$

where $\operatorname{pr}\left(z_{i}\right)$ denotes the parent of $z_{i}$ in the tree. Since the latent variables are meant to capture the hierarchical categorization of the script, we make the assumption that when a higher level script category (for example, $z_{0}$ ) is observed with the actual sequence of events $(x)$, determining the immediate lower level category $\left(z_{1}\right)$ is a deterministic operation. Thus, similar to VQ-VAEs, we parameterize the individual factors of the posterior, $q_{i}\left(z_{i}=k \mid \operatorname{pr}\left(z_{i}\right), x\right)$, as:

$$
\begin{cases}1 & {\mathrm{k}=\operatorname{argmin}_{j}}\left\|f_{i}\left(x, \operatorname{pr}\left(z_{i}\right)\right)-e_{i j}\right\|_{2} \\ 0 & \text { elsewise }\end{cases}
$$

where $f_{i}\left(x, \operatorname{pr}\left(z_{i}\right)\right)$ is an encoding function specific to latent $z_{i}$ and $e_{i j}$ is the $j$ th value em- beddings for $z_{i}$. The distribution $p(x \mid \mathbf{z})$ is similarly parameterized by an decoder function $g\left(\mathbf{z}_{e}\right)$, where $\mathbf{z}_{e}$ is the set of corresponding value embedding for each latent $z_{i}$. We describe the forms of the encoder and decoder in the next section.

\subsection{HAQAE Encoder and Decoder}

During the encoding process, certain parts of the input may provide more evidence towards different parts of the hierarchy. For example, the event (he ate food) gives evidence to the high level category of a restaurant script, while the more specific event (he drank wine) gives more evidence to the lower level category fancy restaurant. Thus during encoding, it makes sense to allow each latent to decide which parts of the input to take into consideration, based on its parent latents. This is accomplished by parameterizing the encoding function for latent $z_{i}$ as an attention over the input $x$, with the parent of $z_{i}$ (more specifically, the embedding for the parent's current value) acting as the 'query' vector. As is standard when using attention, the input sequence of events, $x=\left(x_{1}, \ldots x_{n}\right)$, is first encoded into a sequence of hidden states $\mathbf{h}_{x}=\left(h_{1}, \ldots, h_{n}\right)$ via a RNN encoder. The full encoding function for latent $z_{i}$ can thus be written as:

$$
f_{i}\left(x, \operatorname{pr}\left(z_{i}\right)\right)=\operatorname{attn}\left(\mathbf{h}_{x}, \operatorname{pr}\left(z_{i}\right)\right)
$$

Though any attention formulation is possible, we use the bi-linear attention proposed in Luong et al. (2015) in our implementations. For the root of the latent tree $\left(z_{0}\right)$, which has no parents, we use the averaged value of the encoder vectors $\mathbf{h}_{x}$ as the query vector for its attention.

We can define the decoder in a similar fashion. As is usually done, the distribution $p\left(x \mid \mathbf{z}_{e}\right)$ can be defined in an autoregressive manner using a RNN decoder network. Like the encoding process, different parts of the hierarchy may affect the generation of different parts of the input. We thus also allow the decoder network $g\left(\mathbf{z}_{e}\right)$ to be a RNN with a standard attention mechanism over the latent value embeddings, $\mathbf{z}_{e}$. Since the latent root $z_{0}$ is supposed to capture the highest level information about the script, we use its embedding value, (passed through an affine transformation and tanh activation) to initialize the hidden state of the decoder. Both encoder and decoder can be trained end to end using the same gradient estimation used for VQ-VAE. 


\subsection{Training Objective}

The training objective for HAQAE is nearly the same as the VQ-VAE objective proposed in van den Oord et al. (2017). For a single training example, $x_{i}$ the objective can be written as:

$$
L=-\log p\left(x_{i} \mid \mathbf{z}\right)+\frac{1}{M} \sum_{j}^{M} L_{j}^{R}+\frac{1}{M} \sum_{j}^{M} L_{j}^{C}
$$

where $L_{j}^{R}$ and $L_{j}^{C}$ are the reconstruct and commit loss for the $j$ th latent variable. As in van den Oord et al. (2017), we let $s g(\cdot)$ stand for a stop gradient operator, such that any term passed to it is treated as a constant. The reconstruction loss is defined as:

$$
L_{j}^{R}=\left\|s g\left(f_{j}\left(x, p r\left(z_{j}\right)\right)\right)-e_{j}^{*}\right\|_{2}^{2}
$$

where $e_{j}^{*}$ is the argmin value embedding for $z_{j}$ (for the given input). The reconstruct loss is how the value embeddings for $\mathbf{z}$ are learned, and pushes the value embeddings to be closer to the output of the $f_{i}$. The commit loss is defined as:

$$
L_{j}^{C}=\beta\left\|f_{j}\left(x, p r\left(z_{j}\right)\right)-s g\left(e_{j}^{*}\right)\right\|_{2}^{2}
$$

which forces the encoder to push its output closer to some embedding, preventing a situation in which the encoder maps inputs far away from all embeddings. $\beta$ is a hyperparameter that weighs the commit loss ${ }^{2}$. Note that the commitment loss may be propogated all the way up through the hierarchy of latent nodes. We allow the latent embeddings to receive updates only from the reconstruct and commit loss (not from the NLL loss).

\section{Training Details}

\subsection{Dataset and Preprocessing}

Dataset We use the New York Times Gigaword Corpus as our dataset. The corpus contains a total of around 1.8 million articles. We hold out 4000 articles from the corpus to construct our development (dev) set for hyperparameter tuning and 6000 articles for the test set. The input and output of the model is in the form of an event sequence. Each event is defined as a 4-tuple, $(v, s, o, p)$, containing the verb, subject, object and preposition. Events without prepositions are given a null token in their preposition slot. The components of the events (the verb, subject, etc.) are all taken to be individual tokens, and can thus be treated more or

\footnotetext{
${ }^{2}$ In our implementations we set $\beta=0.25$
}

less like normal text. For example, the events (he played harp), (he touched moon), would be tokenized and given to the model as: played he harp null tup touched he moon null, where null is the null preposition token and tup is a special separation token between events.

We extract event tuples using Open Information Extraction system Ollie (Mausam et al., 2012). We then group together event tuples for 4 subsequent sentences to create a single event sequence. We also ignore tuples with common (is, are, be, ...) and repeating predicates. Finally we have 7123097, 19425, and 28667 event sequences for training, dev, and test dataset respectively. For all the experiments we fix the minimum and maximum sequence lengths to be 8 and 50 respectively.

\subsection{HAQAE Model Details}

The HAQAE model we use across all evaluations uses 5 discrete latent variables, structured in the form of a linear chain (thus no variable has more than one child or parent). Each variable can initially take on $K=512$ values, with all latents having an embeddings dimension of 256. The encoder RNN that performs the initial encoding of the event sequence is a bidirectional, single layer RNN with GRU cell (Cho et al., 2014) with a hidden dimension of 512. The inputs to this encoder are word embeddings derived from the onehot encodings of the tokens in the event sequence. The embeddings size is 300 . We find initializing the embeddings with pretrained GloVe (Pennington et al., 2014) vectors to be useful. The decoder RNN is also a single layer RNN with GRU cells with a hidden dimension of 512 and 300 dimensional (initialized) word embeddings as inputs. For all experiments we use a vocabulary size of 50k. We train the model using Adam (Kingma and $\mathrm{Ba}, 2014$ ) with a learning rate of 0.0005 , and gradient clipping at 5.0. We find that the training converges around 1.5 epochs on our dataset. Further details can be found in our implementation ${ }^{3}$

\subsection{Baselines}

We compare the performance of our proposed model against three previous baselines and a modification of our HAQAE model that removes explicit dependencies between latents.

RNN Language Model For our first baseline system we train a RNN sequence model. This

\footnotetext{
github.com/St onyBrookNLP/HAQAE
} 
model is 2 layered GRU cells with hidden size 512 and embedding size 300 . We use Adam with a learning rate 0.001 . To prevent the problem of exploding gradients, we clip the gradients at 10 . We use uniform distribution $[-0.1,0.1]$ for random initialization and biases initialized to zero. We also use a dropout of 0.15 on the input and output embedding layers but none on the recurrent layers. We initialize the word embedding layer with pretrained Glove vectors as it improved the performance and makes the system directly comparable to HAQAE. We refer to this model as RNNLM in the following sections.

RNNLM + Role Embeddings We also reproduced the model from Pichotta and Mooney (2016) for comparison. This model is similar to the one above except that at each time step the model has an additional role marker input going into it. The marker guides the language model further by indicating what type of input is being currently fed to it: a subject, object, or predicate. These role embeddings are learned during training itself. Hyperparameters are exactly the same as the RNNLN except that the role embeddings have a dimension of 300 . We will refer to this model as RNNLM+Role. We perform hyperparameter tuning of both the models using the development set. We use a vocabulary size of 50k. We trained both the baseline models for 2 epochs on the training set.

VAE We report results using a vanilla VAE model similar to the one used in Bowman et al. (2016). The encoder/decoder for the VAE baseline has the same specs as the encoder/decoder for the HAQAE model, with a latent dimension of 300 . We use linear KL annealing for the first 15000 steps and 0.5 as the word dropout rate.

Hierarchyless HAQAE (NOHIER) In order to test the effect of explicitly having a hierarchy in the latent variables, we additionally train another HAQAE model with no explicit hierarchical latent space. The model still has 5 discrete latent variables like our original model, however each of the variables are independent of each other (given the input). All five variables are have an attention over the input and take the average of encoder vectors $\mathbf{h}_{x}$ as the query vector (as done with the latent root $z_{0}$ in the original model). We additionally designate one of the variables to be used to initialize the hidden state of the decoder. We found the same

\begin{tabular}{c|c|c|c|c}
\multirow{2}{*}{ System } & \multicolumn{2}{|c|}{ validation } & \multicolumn{2}{c}{ test } \\
\cline { 2 - 5 } & NLL & PPL & NLL & PPL \\
\hline RNNLM & 4.52 & 91.84 & 4.51 & 90.92 \\
RNNLM+Role & 4.53 & 92.76 & 4.53 & 92.76 \\
VAE & 4.56 & 95.58 & 4.55 & 94.63 \\
NOHIER & 3.77 & 43.38 & 3.78 & 43.82 \\
HAQAE & $\mathbf{3 . 7 3}$ & $\mathbf{4 1 . 6 8}$ & $\mathbf{3 . 7 4}$ & $\mathbf{4 2 . 1 0}$
\end{tabular}

Table 1: Negative log-likelihood (NLL) and perplexity (PPL) measures on the validation and test set. Lower is better for both metrics.

training hyperparmaters used in the training of the original model to work well here.

\section{Evaluation}

\subsection{Language Modeling: Perplexity}

As our proposed models are essentially language models, it is natural to evaluate their perplexity scores, which can be viewed as an indirect measure of how well the models can identify scripts. We compute per-word perplexity and per-word negative log likelihood on the validation and test sets. We compute these values without considering the end-of-sentence (EOS) token. Table 1 gives these results. A good language model should assign low perplexity (high probability) to the validation and test sets. We observe that HAQAE achieves the minimum negative log likelihood and perplexity scores on both the validation and test sets as compared to the previous RNN-based models. The result is particularly interesting as autoencoders usually perform worse or comparable to other RNN language models in terms of perplexity (negative log likelihood) as is in the case of the vanilla VAE here; similar observations have also been made in Bowman et al. (2016).

\subsection{Inverse Multiple Choice Narrative Cloze}

Narrative cloze evaluations of event based language models (LMs) start with a sequence of events as input and test whether the LMs correctly predict a single held-out event. The standard narrative cloze task has various issues (Chambers, 2017). In our evaluations we opt instead for the multiple choice variant proposed in GranrothWilding and Clark (2016).

One of our goals is to test if our generative model can produce globally coherent scripts by evaluating their ability to generate coherent event sequences. To evaluate this we create a new in- 


\begin{tabular}{c|c|c} 
System & validation & test \\
\hline RNNLM & 25.30 & 26.30 \\
RNNLM+Role & 24.60 & 26.35 \\
VAE & 26.54 & 28.01 \\
NOHIER & 31.68 & $\mathbf{3 4 . 0 0}$ \\
HAQAE & $\mathbf{3 1 . 8 0}$ & 33.85
\end{tabular}

Table 2: Inverse narrative cloze accuracy(\%) on randomly selected $2 \mathrm{k}$ validation and test set. Models scored on whether they assign a higher probability to legitimate event sequence over detractor event sequences. Higher is better.

verse narrative cloze task. Instead of being given an event sequence and predicting a single event to go with it, we instead are given only one event and the model must identify the rest of the event sequence. The model is identifying sequences of events, not single events. We chose this setup because sequences is what we ultimately want, but also because identifying single events resulted in very high scores (around 98\% accuracy). This task proved to be more challenging as an evaluation.

We thus score a model based on the probability it assigns to event sequences that begin with a single input event. A legitimate event sequence should have high probability compared to an event sequence that is stitched together using two random event sequences. We create legitimate event sequences of a fixed length (six) by extracting actual event sequences observed in documents. For every legitimate event sequence, we use the first event in the sequence as a seed event. Then, we construct detractor event sequences for this seed by appending a different sequence of events (number of events being five) from a randomly chosen document. We create five such detractor sequences for every legitimate sequence. We rank the six sequences based on the probabilities assigned by the model and then evaluate the accuracy of the top ranked sequence. A random model will uniformly choose one among the six sequences and thus will score $16=16.60 \%$ on the task. We report results averaged over 2000 sets of legitimate and detractor sequences.

Results in Table 2 show that the HAQAE is substantially better than both RNN LMs and vanilla VAE and similar to the NOHIER model, which shows the usefulness of the quantized embeddings overall as a global representation. The comparable results of the NOHIER model on this task might

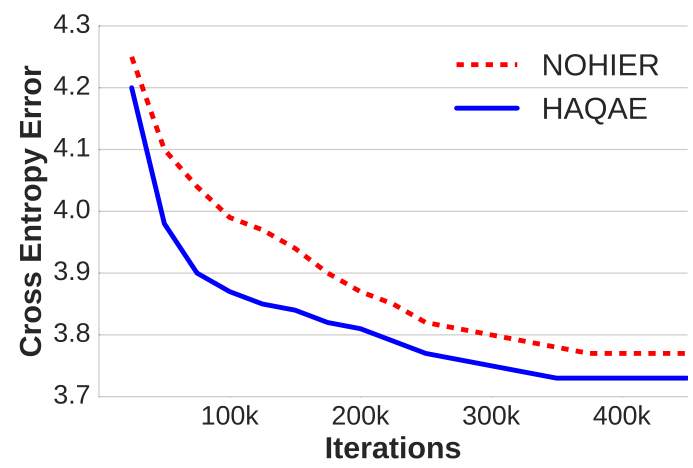

Figure 3: Cross entropy error on dev set of NOHIER (red) and HAQAE (blue) models as training progresses.

also indicate that explicitly modeling the hierarchical structure may not be completely necessary if ones only aim is to capture global coherence. The results on the perplexity task do indicate that overall, modeling the hierarchical structure is useful for better prediction.

\subsection{Comparing HAQAE and NOHIER}

Both HAQAE and NOHIER models achieve the best results across all tasks. The HAQAE model does better on the perplexity task, while results of the two models on the cloze task are nearly the same. One clear benefit of explicitly connecting the latent variables together appears to be in the efficiency of the learning. The HAQAE model performs comparable or better than the NOHIER model despite (in this case at least) having fewer parameters $^{4}$.

The HAQAE model also appears to learn much faster than the NOHIER model. We show this in Figure 3, which shows the per-word cross entropy error on the validation set as training progresses. We observe that the cross entropy error drops much faster in the latter model than the former one. Also, the error is always lower for the HAQAE model.

One possibility is that the NOHIER model learns similar information as the HAQAE model, but due to its lack of explicit inductive bias, takes a longer time to learn this. We leave it as future work to confirm whether this is the case through an in depth study into the properties of the learned discrete latents.

\footnotetext{
${ }^{4}$ NOHIER has more parameters in our case due to each latent taking a bidirectional encoder state as a query vector, as opposed to taking the parent latent vector as the query
} 


\subsection{Evaluating Event Schemas}

So far, we've evaluated how well the models recognize real textual events (perplexity) and how well the models predict events in scripts (narrative cloze). This section evaluates the script generation ability of the model, and specifically its ability to capture hierarchical information with different tracks in scripts (e.g., pleading guilty causes different events to occur than does pleading innocent). In many respects, this section illustrates best the power of HAQAE even though the results are partly subjective.

While we presented two automatic evaluations above, we shift to human judgment to evaluate the scripts themselves. We believe this complements the empirical gains already presented. The scripts generated by the models were shown to human judges and scored on several metrics.

Most previous work on script induction starts with a seed event and then grows the script based on measures of event proximity or from sampling the distribution with the seed as context. While effective in generating a bag of events, a major problem in all previous work is that conflicting events are included (sentenced and acquitted). While the events are related and part of the same high-level script, they should never appear together in an actual instance of a script.

In order to evaluate our model for this type of knowledge, we instead defined a seed as 2 events: the first event sets the general topic, and the second event starts a specific track in that topic. For instance, below are two seeds that are intended to generate two tracks for the same script:

$$
\begin{aligned}
& \text { "people reported fire" } \\
& \text { "fire spread in neighborhood" } \\
& \text { "people reported fire" } \\
& \text { "fire spread to forest" }
\end{aligned}
$$

For each seed ( 2 events in one seed) we select the first 3 events generated by a model conditioned on the seed as context. The 2 events in a seed thus initialize the latent variable values, which then inform the decoder to generate more events (we choose the first 3 ). The strength of our model is that the second event helps select the more specific script track, and to ignore conflicting events in other tracks.

While generating for both RNNLM+Role and HAQAE models, we additionally enforce a constraint that restricts models from generating events that have a predicate that has already been generated, as well as events whose subject and object are the same.

We evaluated the RNNLM+Role model from Pichotta and Mooney (2016) against our proposed HAQAE. Each model was given 40 seeds ( 20 first event each with 2 contrasting second seed) and thus generated 40 scripts. The annotators were also shown the seeds ( 2 events), and then asked to rate each three-event sequence for various metrics described below.

Non-Sensical (Sense): Binary, is each event itself non-sensical or understandable?

Event Relevance (Rel): Binary, each event was scored for being relevant or not to the script topic. This ignored whether it was consistent with the seed's branch.

Coherency with Branch (Coh): 0-2, each event was scored for being coherent with the seed's specific branch (the second event). 0 means not at all, 1 means somewhat, and 2 means yes.

Branching Uniqueness (BranchU): 0-2, each pair of scripts (both branches of the same topic) were scored for overlap of events. 0 means similar events generated for both, 1 means some similar events, and 2 means distinct. This score is important because some RNN decoders might ignore the second event and focus on the general topic only.

Branching Quality (BranchQ): 0-2, each generated branch was scored for branch quality. 0 means the generated events are not specific to the branch, 1 means some are specific, and 2 means most/all events are specific. This is the most important score in measuring how well a model captures hierarchical structure and script tracks.

Two expert annotators evaluated the generated event sequences. In case of disagreements in scores, we also involved a third annotator to resolve these conflicts. Results for this task are shown in Table 3.

Both RNNLM and HAQAE produce sensical events, but the HAQAE model outperforms on all other metrics. It produces more relevant and coherent events for the topic at hand (relevance and coherency). But most important to the goals of this paper, it doubles the RNNLM scores on branching uniqueness and quality. This is because an RNNLM mostly generates from a bag of events after encoding the seed, but the HAQAE utilizes its latent space to produce branch-specific tracks of event sequences. Tables 4 show a few such ex- 


\begin{tabular}{c|c|c|c|c|c} 
System & Sense $(\%)$ & $\operatorname{Rel}(\%)$ & $\operatorname{Coh}(0-2)$ & BranchU(0-2) & BranchQ(0-2) \\
\hline RNNLM+Role & 93.13 & 84.73 & 1.35 & 0.65 & 0.51 \\
HAQAE & 93.13 & 95.37 & 1.59 & 1.35 & 1.00
\end{tabular}

Table 3: Human evaluation of schemas generated for the seed events. HAQAE consistently performs better than the baseline model. Higher is better for all metrics.

\begin{tabular}{|l|l|l|}
\hline RNN+Role & $\begin{array}{l}\text { bomb found in backpack, bomb failed to detonate } \\
\text { bomb found in backpack, bomb detonated } \\
\text { people reported fire, fire spread to forest } \\
\text { people reported fire, fire spread to neighborhood }\end{array}$ & $\begin{array}{l}\text { bomb killed people, bomb detonated in blast } \\
\text { explosion killed people, people injured in blast } \\
\text { fire destroyed building } \\
\text { fire damaged building }\end{array}$ \\
\hline \hline HAQAE & $\begin{array}{l}\text { bomb found in backpack, bomb failed to detonate } \\
\text { bomb found in backpack, bomb detonated } \\
\text { people reported fire, fire spread to forest } \\
\text { people reported fire, fire spread to neighborhood }\end{array}$ & $\begin{array}{l}\text { they found evidence, explosive hidden in luggage } \\
\text { blast left crater, blast killed people } \\
\text { fire burned acres } \\
\text { fire destroyed building }\end{array}$ \\
\hline \multicolumn{2}{|l}{} \\
\hline
\end{tabular}

Table 4: Sample outputs from the baseline and our proposed system. The seeds (what is given to the system) are shown in the left column while the outputs are on the right. HAQAE is able to distinguish between the contrasting seeds. Red highlights the lack of branching quality in the baseline model and Blue highlights the correct behavior as exhibited by HAQAE.

\begin{tabular}{|l|}
\hline person denied charges, lawsuit filed by person, judge dismissed lawsuit \\
person denied charges, they accused person, person resigned in january \\
\hline clinton carried promises, clinton began in 1988, clinton made changes \\
campaign carried promises, campaign began on september, campaign made effort \\
\hline campaign carried promises, campaign began on september, campaign made effort \\
team carried for championship, team played in philadelphia, they won champoinship \\
\hline
\end{tabular}

Table 5: Results of changing a single latent variable while keeping others fixed. Lower level latents typically change ending/beginnings or entity names (Rows 1 and 2). The top level latent changes the topic and may occasionally preserve the form (Row 3 )

amples.

\subsection{Observations about the Latent Variables}

We also look at how changing the values of various latent variables change the resulting output, in order to get a small idea as to what properties the variables capture. We find that the root level variable $z_{0}$ has the largest effect on the output, and typically corresponds to the domain that the sequence of events belong to. The non root variables generally change the output on a smaller scale, however we find no correspondence between the level of the variable and the amount of output that is affected upon changing its value.

One reason for the difficulty of interpreting the variables is that the model conditions on them through attention, thus changing the value of one does not necessarily need to have any effect.

We do find that changing the lower level latents generally leads to the ending/beginning of the sequence changing or the entities of the sequence changing (but still remaining in the same topical domain). We additionally find that changing the top level latent may often preserve the overall form of the event sequence, and only transform the topic. We provide examples of these output by our system in Table 5.

\section{Related Work}

Scripts were originally proposed by Schank and Abelson (1975) and further expanded upon in Schank and Abelson (1977). The notion of hierarchies in scripts has been studied in the works of Abbott et al. (1985) and Bower et al. (1979). Mooney and DeJong (1985) present an early non probabilistic system for extracting scripts from text. A highly related work by Miikkulainen (1990) provides an early example of a system explicitly designed to take advantage of the hierarchical nature of scripts, creating a model of scripts based on self organizing maps (Kohonen, 1982). Interestingly, self organizing maps also utilize vector quantization during learning (albeit in a different way than done here).

Recent work starting from Chambers and Jurafsky (2008) has focused on learning scripts as 
prototypical sequences of events using event cooccurrence. Further work has framed this task as a language modeling problem (Pichotta and Mooney, 2016; Rudinger et al., 2015; Peng and Roth, 2016). Other work has looked at learning more structured forms of script knowledge called schemas (Chambers, 2013; Balasubramanian et al., 2013; Nguyen et al., 2015) which focuses on additionally inducing script specific roles to be filled by entities. In this work we treat event components as separate tokens, though work has also looked into methods for composing this components into a single distributed event representation (Modi and Titov, 2014; Modi, 2016; Weber et al., 2018). We leave this as possible future work.

The hierarchical structure of our proposed model is similar to structure of the latent space in other VAE variants (Sonderby et al., 2016; Zhao et al., 2017), with the discrete variables and attentions in our model being the major differences. $\mathrm{Hu}$ et al. (2017) present a VAE based model for controllable text generation, with different latents controlling different aspects of the generated text, but requiring labels for semi-supervision. Other methods using discrete variables for VAEs have also been proposed (Rolfe, 2017), as have variations in the VQ-VAE learning process (Sonderby et al., 2017)

\section{Conclusion}

We proposed a new model, HAQAE, for script learning and generation that is one of the first to model the hierarchy that is inherent in this type of real-world knowledge. Previous work has focused on modeling event sequences with language models, while ignoring the problem of contradictory events and different tracks being jumbled together. The hierarchical latent space of HAQAE instead attends to the choice points in event sequences, and is able to provide some discrimination between tracks of events.

While HAQAE is motivated by the specific need for hierarchies in scripts, it can also be seen as a general event language model. As a language model HAQAE has a substantially lower perplexity on our test set than previous RNN models despite HAQAE's decoder having fewer parameters.

We also presented a new inverse narrative cloze task that is a multiple-choice selection of event sequences. It proved to be a very difficult task with systems producing accuracies in the mid $20 \%$ range. HAQAE and NOHEIR were the only systems to break 30 with a top accuracy of $34.0 \%$. This further illustrates that using a latent space to capture script differences helps identify relevant sequences.

To our knowledge, all previous work on script induction has focused on learning single event sequences or bags of events. We view our proposed model as a new step toward learning different details about scripts, such as tracks and hierarchies. Though the proposed model works well empirically, understanding exactly what is learned in the latent variables is non trivial, and is a possible direction for future work.

\subsection{Acknowledgements}

This work is supported in part by the National Science Foundation under Grant IIS-1617969.

\section{References}

Valerie Abbott, John B Black, and Edward E Smith. 1985. The representation of scripts in memory. Journal of Memory and Language, 24(2):179 - 199.

Niranjan Balasubramanian, Stephen Soderland, Mausam, and Oren Etzioni. 2013. Generating coherent event schemas at scale. In EMNLP, pages 1721-1731.

Gordon H Bower, John B Black, and Terrence J Turner. 1979. Scripts in memory for text. Cognitive psychology, 11(2):177-220.

Samuel R. Bowman, Luke Vilnis, Oriol Vinyals, Andrew M. Dai, Rafal Jozefowicz, and Samy Bengio. 2016. Generating sentences from a continuous space. CoNLL.

Nathanael Chambers. 2013. Event schema induction with a probabilistic entity-driven model. In Proceedings of the 2013 Conference on Empirical Methods in Natural Language Processing, pages 17971807.

Nathanael Chambers. 2017. Behind the scenes of an evolving event cloze test. In Proceedings of the $2 n d$ Workshop on Linking Models of Lexical, Sentential and Discourse-level Semantics, pages 41-45.

Nathanael Chambers and Dan Jurafsky. 2008. Unsupervised learning of narrative event chains. Proceedings of ACL-08: HLT, pages 789-797.

Kyunghyun Cho, Bart van Merriënboer, Çalar Gülçehre, Dzmitry Bahdanau, Fethi Bougares, Holger Schwenk, and Yoshua Bengio. 2014. Learning phrase representations using rnn encoder-decoder for statistical machine translation. In Proceedings of 
the 2014 Conference on Empirical Methods in Natural Language Processing (EMNLP), pages 17241734, Doha, Qatar. Association for Computational Linguistics.

Mark Granroth-Wilding and Stephen Clark. 2016. What happens next? event prediction using a compositional neural network model. In $A A A I$, pages 2727-2733.

Zhiting Hu, Zichao Yang, Xiaodan Liang, Ruslan Salakhutdinov, and Eric P. Xing. 2017. Toward controlled generation of text. ICML.

Diederik P Kingma and Jimmy Ba. 2014. Adam: A method for stochastic optimization. arXiv preprint arXiv:1412.6980.

Diederik P Kingma and Max Welling. 2014. Autoencoding variational bayes. ICLR.

Teuvo Kohonen. 1982. Self-organized formation of topologically correct feature maps. Biological cybernetics, 43(1):59-69.

Thang Luong, Hieu Pham, and Christopher D. Manning. 2015. Effective approaches to attention-based neural machine translation. In EMNLP, pages 1412-1421. The Association for Computational Linguistics.

Mausam, Michael Schmitz, Stephen Soderland, Robert Bart, and Oren Etzioni. 2012. Open language learning for information extraction. In Proceedings of the 2012 Joint Conference on Empirical Methods in Natural Language Processing and Computational Natural Language Learning, pages 523-534. Association for Computational Linguistics.

Risto Miikkulainen. 1990. Script recognition with hierarchical feature maps. Connection Science, 2:83101.

Ashutosh Modi. 2016. Event embeddings for semantic script modeling. In Proceedings of the Conference on Computational Natural Language Learning (CoNLL), Berlin, Germany.

Ashutosh Modi and Ivan Titov. 2014. Inducing neural models of script knowledge. In Proceedings of the Eighteenth Conference on Computational Natural Language Learning, pages 49-57, Ann Arbor, Michigan. Association for Computational Linguistics.

Raymond Mooney and Gerald DeJong. 1985. Learning schemata for natural language processing. In Ninth International Joint Conference on Artificial Intelligence (IJCAI), pages 681-687.

Kiem-Hieu Nguyen, Xavier Tannier, Olivier Ferret, and Romaric Besançon. 2015. Generative event schema induction with entity disambiguation. In ACL, pages 188-197.
Aaron van den Oord, Oriol Vinyals, and Koray Kavukcuoglu. 2017. Neural discrete representation learning. NIPS.

Haoruo Peng and Dan Roth. 2016. Two discourse driven language models for semantics. In Proceedings of the 54th Annual Meeting of the Association for Computational Linguistics, ACL 2016, August 712, 2016, Berlin, Germany, Volume 1: Long Papers. The Association for Computer Linguistics.

Jeffrey Pennington, Richard Socher, and Christopher Manning. 2014. Glove: Global vectors for word representation. In EMNLP, pages 1532-1543.

Karl Pichotta and Raymond J Mooney. 2016. Learning statistical scripts with lstm recurrent neural networks. In $A A A I$, pages 2800-2806.

Jason Tyler Rolfe. 2017. Discrete variational autoencoders. ICLR.

Rachel Rudinger, Pushpendre Rastogi, Francis Ferraro, and Benjamin Van Durme. 2015. Script induction as language modeling. In Proceedings of the 2015 Conference on Empirical Methods in Natural Language Processing, pages 1681-1686.

Roger C Schank and Robert P Abelson. 1975. Scripts, plans, and knowledge. In IJCAI.

Roger C Schank and Robert P Abelson. 1977. Scripts, plans, goals and understanding: An inquiry into human knowledge structures. 1977. Mhwah, NJ (US): Lawrence Erlbaum Associates.

Casper Kaae Sonderby, Ben Poole, and Andriy Mnih. 2017. Continuous relaxation training of discrete latent variable image models. NIPS Bayesian Deep Learning Workshop.

Casper Kaae Sonderby, Tapani Raiko, Lars Maale, Sren Kaae Snderby, and Ole Winther. 2016. Ladder variational autoencoders. NIPS.

Noah Weber, Niranjan Balasubramanian, and Nathanael Chambers. 2018. Event representations with tensor-based compositions. AAAI, abs/1711.07611.

Shengjia Zhao, Jiaming Song, and Stefano Ermon. 2017. Learning hierarchical features from deep generative models. In Proceedings of the 34th International Conference on Machine Learning, ICML 2017, Sydney, NSW, Australia, 6-11 August 2017, volume 70 of Proceedings of Machine Learning Research, pages 4091-4099. PMLR. 